\title{
PheniX: a new vision for the hard X-ray sky
}

\author{
Jean-Pierre Roques • Elisabeth Jourdain • Loredana Bassani • \\ Angela Bazzano $\cdot$ Renaud Belmont • A. J. Bird · E. Caroli • M. Chauvin • \\ D. Clark • N. Gehrels • U. Goerlach • F. Harrisson • P. Laurent • J. Malzac • \\ P. Medina · A. Merloni $\cdot$ S. Paltani • J. Stephen • P. Ubertini · J. Wilms
}

Received: 16 May 2011 / Accepted: 27 June 2011 / Published online: 2 August 2011

(c) Springer Science+Business Media B.V. 2011

\begin{abstract}
We are proposing a mission devoted to high energy X-ray astronomy that is based on a focusing telescope operating in the 1-200 keV energy range but optimized for the hard X-ray range. The main scientific topics concern: Physics of compact objects: The proximity of compact objects provides a unique laboratory to study matter and radiation in extreme conditions of temperature and density in strong gravitational environment. The emission of
\end{abstract}

J.-P. Roques $(\bowtie) \cdot$ E. Jourdain $\cdot$ R. Belmont · M. Chauvin · D. Clark · J. Malzac IRAP, Universite de Toulouse/CNRS, 9 Avenue du Colonel Roche, BP44346,

31028 Toulouse Cedex 4, France

e-mail: roques@cesr.fr

L. Bassani · E. Caroli · J. Stephen

Istituto di Astrofisica Spaziale e Fisica Cosmica, INAF-IASF Bologna, Area Della Ricerca Cnr, via Gobetti 101, 40129 Bologna, Italy

A. Bazzano · P. Ubertini

INAF, IASF Sezione di Roma, Via Fosso del Cavaliere 100, Tor Vergata, IT 00133 Rome, Italy

\footnotetext{
A. J. Bird

University of Southampton, School of Physics \& Astronomy, University of Southampton, Highfield, Southampton, SO17 1BJ, UK

N. Gehrels

NASA/GSFC, Code 661, Astroparticle Physics Laboratory, Greenbelt, MD 20771, USA

P. Laurent

Laboratoire Astroparticule et Cosmologie, Batiment Condorcet, 10, rue Alice Domont et Léonie Duquet, 75205, Paris Cedex 13, France

P. Laurent

IFU/Service d'Astrophysique, Bat. 709 Orme des Merisiers CEA Saclay,

91191 Gif-sur-Yvette Cedex, France
} 
high energy photons from these objects is far from being understood. The unprecedented sensitivity in the high energy domain will allow a precise determination of the non-thermal processes at work in the vicinity of compact objects. The full 1-200 keV energy coverage will be ideal to disentangle the emission processes produced in the spacetime regions most affected by strong-gravity, as well as the physical links: disk-thermal emission-iron line-comptonisationreflection-non-thermal emission-jets. Neutron stars-magnetic field-cyclotron lines: Time resolved spectroscopy (and polarimetry) at ultra-high sensitivity of AXP, milliseconds pulsars and magnetars will give new tools to study the role of the synchrotron processes at work in these objects. Cyclotron lines-direct measurement of magnetic filed-equation of state constraints-short bursts-giant flares could all be studied with great details. AGN: The large sensitivity improvement will provide detailed spectral properties of the high energy emission of AGN's. This will give a fresh look to the connection between accretion and jet emission and will provide a new understanding of the physical processes at work. Detection of high-redshift active nuclei in this energy range will allow to introduce an evolutionary aspect to highenergy studies of AGN, probing directly the origin of the Cosmic X-ray Background also in the non-thermal range $(>20 \mathrm{keV})$. Element formationSupernovae: The energy resolution achievable for this mission $(<0.5 \mathrm{keV})$ and a large high energy effective area are ideally suited for the 44Ti line study (68 and $78 \mathrm{keV}$ ). This radioactive nuclei emission will give an estimate of their quantities and speed in their environment. In addition the study of the spatial structure and spectral emission of SNR will advance our knowledge of the dynamics of supernovae explosions, of particles acceleration mechanisms and how the elements are released in the interstellar medium. Instrumental design: The progress of X-ray focusing optics techniques allows a major step in the instrumental design: the collecting area becomes independent of the detection area. This drastically reduces the instrumental background and will open a new era. The optics will be based on depth-graded multi-layer mirrors in a Wolter

A. Merloni

Max-Planck-Institut für extraterrestrische Physik, Excellence Cluster Universe, TUM,

Boltzmannstr. 2, 85748 Garching, Germany

S. Paltani

INTEGRAL Science Data Center, Geneva Observatory, 16 Chemin Ecogia, CH 1290 Versoix, Switzerland

J. Wilms

University of Erlangen-Nuremberg, Schlossplatz 4, 91054 Erlangen, Germany

U. Goerlach · P. Medina

Institut Pluridisciplinaire Hubert Curien, 23, rue du Loess, BP28, 67037 Strasbourg Cedex 2,

France

F. Harrisson

Space Radiation Lab, 290-17 Caltech, Pasadena, CA 91125, USA 
I configuration. To obtain a significant effective area in the hundred of $\mathrm{keV}$ range a focal length in the 40-50 meters range (attainable with a deployable mast) is needed. In addition such a mission could benefit from recent progress made on mirror coating. We propose to cover the 1-200 keV energy range with a single detector, a double-sided Germanium strip detector operating at $80 \mathrm{~K}$. The main features will be: (a) good energy resolution $(.150 \mathrm{keV}$ at $5 \mathrm{keV}$ and $<.5 \mathrm{keV}$ at $100 \mathrm{keV}$ ), (b) 3 dimensional event localization with a low number of electronic chains, (c) background rejection by the 3D localization, (d) polarisation capabilities in the Compton regime.

Keywords Hard X-rays • Germanium • Grazing incidence optics • Space instrument

\section{Scientific objectives}

\subsection{Introduction}

Learning about the physical conditions in the environment of a black hole $(\mathrm{BH})$ is of fundamental importance for at least two reasons: first, the existence of black holes is the most far-reaching implication of the theory of general relativity (GR). Indeed astrophysical black holes represent an opportunity to observe signatures of GR in strong gravitational field. Second, black holes are present in the nuclei of probably all galaxies. The evolution of black holes and galaxies appear intrinsically linked. Understanding how black holes interact with their environment is a prerequisite to the study of the formation and evolution of the first structures of the universe.

A black hole may become visible when it accretes gas from its environment and then radiates a fraction of the gravitational energy of the infalling material. Due to viscosity, the infalling gas loses its angular momentum and forms an accretion disc. In the innermost part of the accretion flow, observations indicate the presence of very hot plasma, with a temperature of a few billion Kelvin, leading to copious non-thermal X-ray emission. The nature of this hot plasma (that is usually called the 'corona') remains controversial. It could be a form of hot fully ionized viscously heated accretion flow (e.g. ADAF; [24]) or a strongly magnetized accretion disc corona (analogous to the solar corona). Another possibility would be that this corona could be the base of a jet. Indeed, those relativistic jets are usually observed in the radio band but can, at least occasionally, contribute significantly to the X-ray emission. Although we have clear observational evidences of the existence of all these distinct physical components, we still lack a full understanding of how the gravitational energy is converted into radiation and jet kinetic power through accretion.

Stellar mass objects $(\mathrm{M} \sim 10 \mathrm{Msun})$ are formed at the end of the life of a massive star. If they are formed in a binary system they can accrete the gas from their companion star and are then observed as X-ray binaries. On the other hand, super-massive black holes (SMBH) are located at the centre of galaxies 
with masses larger than a million times the mass of the sun. When these black holes are accreting, they appear as Active Galactic Nuclei (AGN). In addition, Ultraluminous X-ray sources (ULXs) appear as very bright point-like extragalactic sources offset from the nuclei of their host galaxy. Some ULX's appear to be stellar mass objects accreting at an extremely high rate, others are intermediate mass Black holes (with mass $10^{2}-10^{4}$ Msun). The observations of all these astrophysical sources allow us to probe the physical conditions close to black holes over a wide range of accretion regimes. For the astrophysicist, the main questions are the structure and geometry of the accretion flow, as well as its evolution as a function of mass accretion rate, mass and spin of the black hole. In the case of AGN, we also want to understand the joint evolution of the black hole and host galaxy as a function of redshift.

Neutron stars are another possible product of the gravitational collapse of a massive star. If a neutron star is formed in a binary system it may accrete in a way similar to black hole X-ray binaries. It then produces similar observable features such as an accretion disc, X-ray corona and jets. There are however two main observable differences between black holes and neutron stars: the presence, in neutron stars, of a hard surface and a very strong magnetic field (up to $\sim 10^{15} \mathrm{G}$ ). Even when the neutron star is not accreting, it can produce large amounts of radiation (in particular at high energies) powered by the rotation of the neutron star (spin powered pulsars) or decay of the strong magnetic field (magnetars). Neutron stars provide a unique laboratory to study physics under extreme condition: matter at nuclear densities, huge magnetic fields, and strong gravity. The comparison of the properties of accreting black holes and neutron stars is also useful to understand accretion processes.

Another topic related to compact objects concerns the production of heavy elements. Part of them is created through explosive events like those giving birth to neutron stars (supernovae). Supernova remnants (SNR) are thus ideal targets to understand how these elements are released in the interstellar medium, as well as to study the dynamics of supernovae explosions. Heavy element production is revealed thanks to specific lines and extraction of all the relevant information will rely on the powerful spectroscopic capability.

Among the other objects for which an excellent sensitivity in the Hard XRays and a very wide energy coverage will be very useful, we have to mention the high mass X Ray Binaries, the magnetic Cataclysmic Variables (binary system containing a white dwarfs) which are very few to be detected above $10 \mathrm{keV}$ and the galaxy clusters. In all cases, the detection of theses faint non-thermal hard X-ray emissions would represent a big step forward in the understanding of the processes at work. In the following we develop in more details the advances we can expect on the main issues from a future hard X-ray mission.

\subsection{Accretion and ejection flows around Black Holes}

As described above, the accretion/ejection flow around a black hole presents three main physical components (the accretion disc, the corona and the jet) 
which can all contribute to the X-ray emission. In the immediate vicinity of the central source, the three components are dynamically coupled, and vary simultaneously: they have to be studied together in order to understand the whole picture of black hole accretion. The innermost part of the accretion flow cannot be imaged. We rely essentially on spectroscopy to figure out its structure. In order to disentangle the different spectral components and follow their evolution, it is crucial to cover a range in energy that is as broad as possible.

These sources show strong variability on all time scales (down to the millisecond in black hole binaries). The study of this variability provides crucial information on the dynamical processes in the vicinity of the black hole. By lack of sensitivity above $10 \mathrm{keV}$, timing studies in the hard X-ray band are currently limited to a couple of very bright sources. X-ray polarisation is potentially another important tool that could be used to extract valuable information about the geometry of the inner part of the accretion flow and the structure of the magnetic field but none of the current X-ray telescopes have polarimetric capability.

An instrument providing simultaneously accurate measurements of the Xray spectra and polarisation over a large band (1-200 keV at least) is badly needed to better understand the corona, accretion disc and jet and their interconnections.

\subsubsection{Investigating the nature of the corona}

As mentioned above, it is not clear whether the corona is constituted by a hot accretion flow, a magnetically dominated accretion disc corona, or the jet itself. The physical structure and geometry of this relativistic plasma appear to change with the various accretion regimes but we do not understand how and why. Moreover, the properties of the corona seem to be intimately connected to that of the radio jet, but it is not clear which role (if any) it plays in the jet formation process. By modelling the broadband X-ray spectra one can determine the nature of the electron distribution (i.e. thermal vs non-thermal). When the plasma is thermal, one can measure physical parameters such as the Thomson optical depth of the plasma or the electron temperature. This however requires sensitivity around the cut-off energy $(\sim 100 \mathrm{keV})$. Studying the shape of the electron distribution in the corona provides in turn constraints on the particle acceleration, heating and thermalisation processes.

Finally, the Comptonisation process imprints weak, energy dependent, polarisation features (a few percents) on the emerging spectra. These polarisation features depend on the relative geometry of the incident photons and scattering electrons (see Fig. 1, and [29]). If we could measure them, polarisation and spectral information could then be combined to put strong constraints on the properties of the corona.

Polarisation can also be used to test for the contribution of highly polarised jet synchrotron radiation to the hard X-ray spectra. In fact, the availability of a spectropolarimeter sensitive over a broad energy band would open a whole new window on these sources. 
Fig. 1 Polarization degree expected from a slab corona for different height/radius ratios [29]

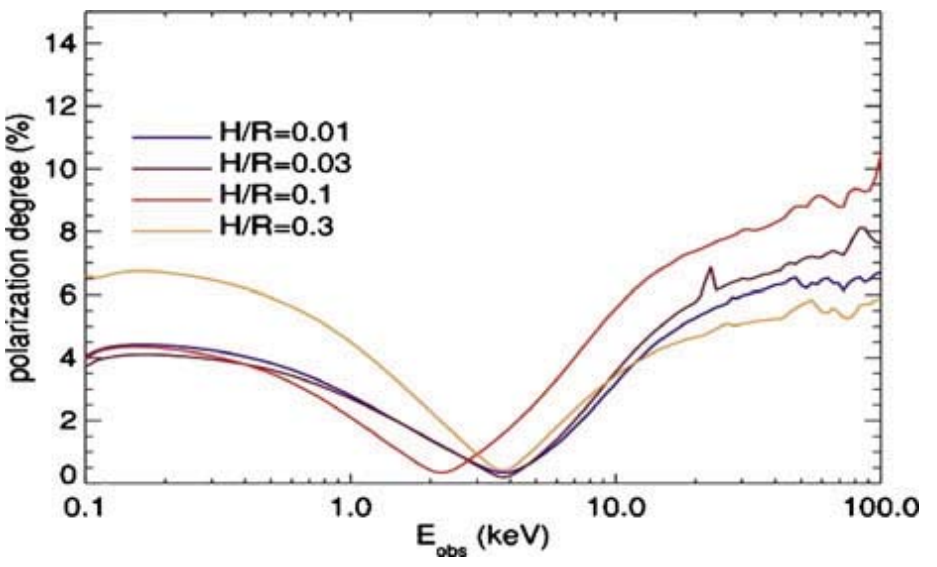

A peculiar object will deserve more attention: the emission of the Galactic central supermassive black hole remains a big challenge by itself due to its low luminosity quiescent level in the hard X-ray domain coupled with an X-ray flaring activity. In a more general way, exploring the spectral evolution of this and other very faint sources requires a highly sensitive broadband instrument.

Making significant progress on the understanding of the corona at higher luminosities now requires time resolved spectro-polarimetry over a large band.

In a few bright hard state X-ray binaries, GRANAT, OSSE and INTEGRAL have detected a high energy excess with respect to thermal Comptonisation models. In GX-339-4 the excess was clearly detected by INTEGRAL in the 100-200 keV band and shown to be variable on time scales of a few hours [11]. The nature of this excess is not clear and the magnetic field can play a major role. However, the observed coronal temperatures are rather high; this requires that the magnetic field is relatively low (i.e. strongly subequipartition). Such a low magnetic field would have important consequences for any dynamical coronal model. It is therefore important to investigate the nature of this non-thermal excess both through detailed spectral/polarisation modeling and variability studies. A gain in sensitivity of a factor of 100 with respect to INTEGRAL will allow detecting such an excess with an exposure as short as a few minutes.

In the soft states, although the accretion rate may be high, the coronal emission can be weak and difficult to measure. In fact, the source sometimes reaches an ultra soft state in which the coronal emission is barely detectable with current (and previous) instruments. Previous investigations in the soft $\mathrm{X}$-ray band indicate that the slope of the coronal spectrum changes when a source evolves toward softer spectra. In these disc dominated states the detailed modeling of the thermal disc emission is used to infer the black hole spin (see e.g. [25] and Section 2). As the coronal emission may contaminate the disc spectrum, it is important to understand how the corona evolves in this case. This requires sensitive measurements of the global evolution in the $\mathrm{X}$ and hard X-ray bands. 
Finally, it is currently not clear what triggers the spectral state transitions and what is really happening in the accretion/ejection flow during these events. The sensitivity currently achieved by the X-rays instruments has to be extended toward higher energies in order to study in detail the variability on different time scales. In particular this would make it possible to follow the rapid variation of the spectrum occurring during state transitions and to track simultaneously and in exquisite details the evolution of the accretion disc and corona.

\subsubsection{The accretion disc: iron line and reflection features}

The distribution of black hole spins in supermassive black holes (SMBH) contains information about the history of the growth of black hole in the universe with significant implications for cosmological models (see e.g. [33]). This is particularly important in the context of the formation of jets since some models [6] predict that the jet strength should depend on the spin of the black hole.

Measuring this distribution of spins is therefore an important goal. In addition the black hole spin may have an effect on the physics of the accretion flow that we would like to understand. A possible measure of the black hole spin can be obtained from reprocessing and reflection features produced by the coronal hard X-ray emission impinging on the accretion disc (see Figs. 2 and 3). Indeed, the spectral shape of the iron $\mathrm{K} \alpha$ line (around $6.4 \mathrm{keV}$ ) and reflection components can be strongly altered (broadened) by special and general relativistic effects. In particular the line profile is very sensitive to the spin of the black hole. Such relativistically broadened iron lines have been observed in many sources (both in AGN and BH binaries, see e.g. [12]). However, the iron $\mathrm{K} \alpha$ line profile is very sensitive to the shape of the underlying continuum which can be measured only with broad band instruments that are sensitive above $10 \mathrm{keV}$. Moreover the information above $10 \mathrm{keV}$ is crucial to measure and model the reflection component together with iron line.

In order to solve this issue we require a unique high throughput detector covering the whole (1-200 keV) energy range, accepting counts rate as high as a few Crab.

In sources in which the emission is very concentrated close to the black holes, general relativistic effects can strongly beam the coronal emission toward the disc (light bending, see e.g. [23]) increasing the reflected component contribution. However, in the soft X-ray band these spectra can be fitted equally well with models in which the source is not dominated by reflection but seen through a complex absorber ([32], and see Fig. 3).

Again discriminating between these models will require a larger sensitivity at high energy around the peak of the reflection bump. Only instruments with a broad energy band coverage and sensitivity will allow breaking down the degeneracy between these models. The study of the reflected features in polarisation could also be used jointly with spectral and variability studies in order to map the innermost parts of the accretion flow. 
Fig. 2 The broadband X-ray spectrum of Cygnus X-1 obtained by Suzaku (from [21]) with different models. The three different Suzaku instruments (XIS/PIN/GSO) are fitted simultaneously in the different energy bands. During the fit procedure the relative normalization is kept as a free parameter to allow for cross calibration uncertainties

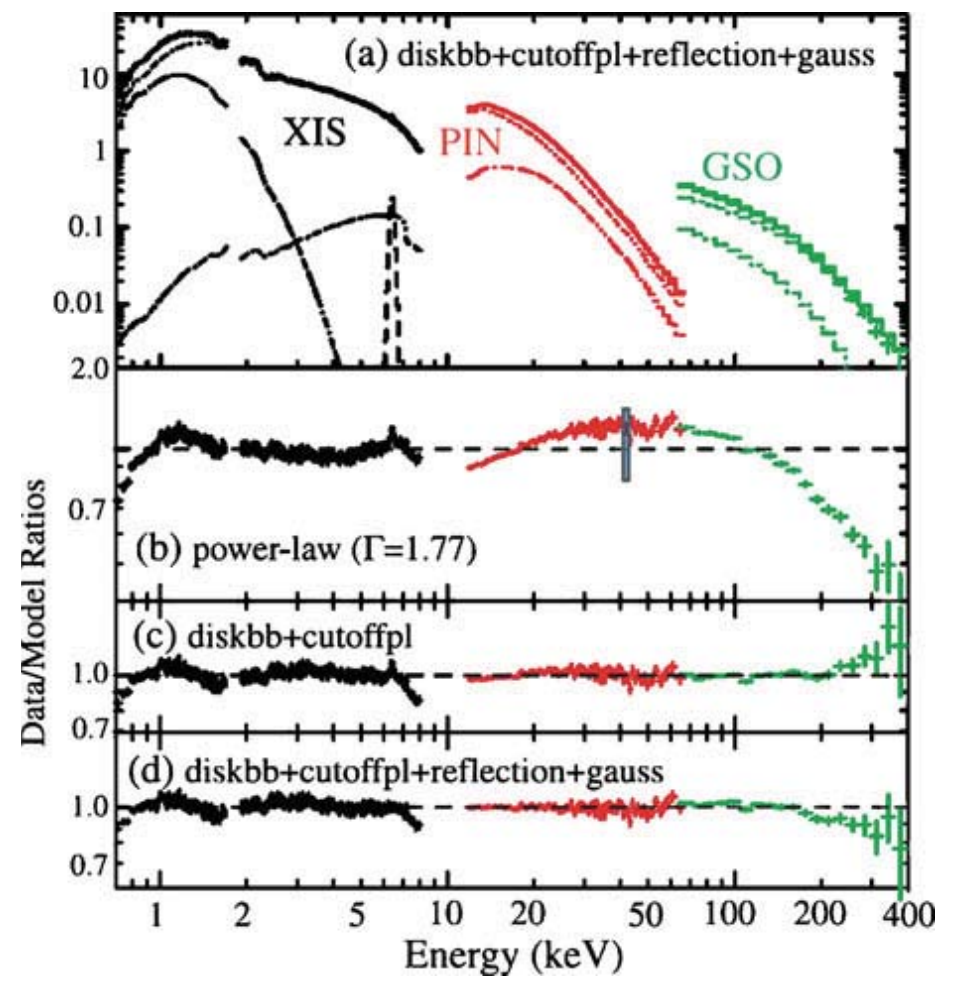

\subsubsection{Studying the most extreme behavior in jet dominated $X$-ray sources}

In Blazars, the source emission appears dominated by the jet. The spectral energy distributions (SEDs) of these objects are characterized by two broad peaks. The first one, ascribed to incoherent synchrotron emission, occurs anywhere from IR to X-rays, and the second, due to Compton scattering or another more energetic synchrotron component, falls in the $\mathrm{MeV}-\mathrm{GeV}$ band [14]. No reprocessing features of the type seen in Seyfert galaxies are observed.

Fig. 3 Best fit models to the XMM spectra of Mkr 841. The thick solid and dashed curves show the relativistically blurred reflection model and absorption model spectra respectively. Over-plotted in thin lines are the different components of each model. From Petrucci et al. [27]

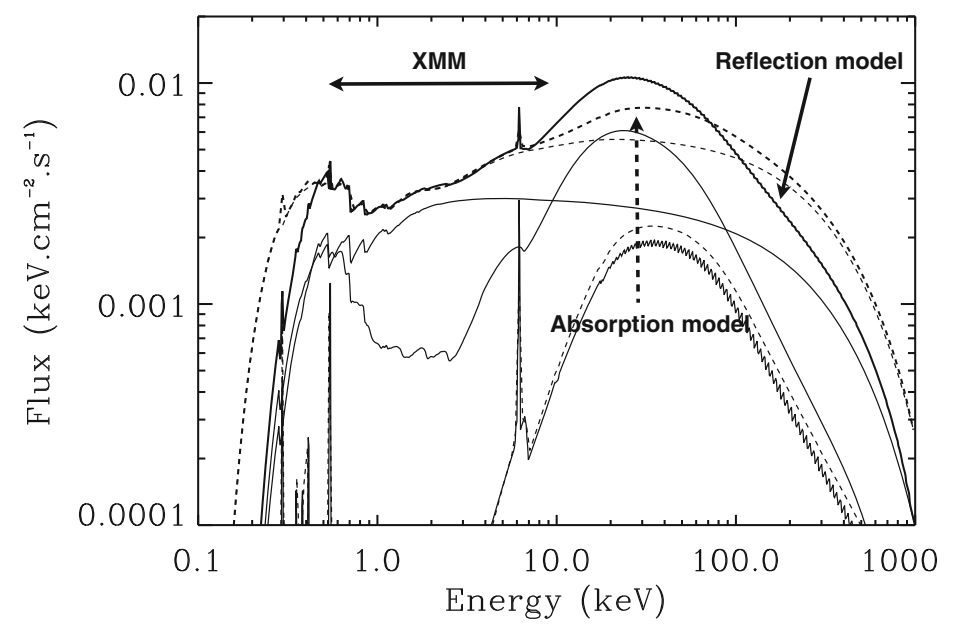


Within the Blazar population there are sub-classes of objects which are prominent in hard X-rays [15].

These are at the extremes of the Blazar sequence. At one end, we find objects having very small jet power and no sign for thermal disk emission: they are the $\mathrm{TeV}$ emitting BL Lacs having a synchrotron peak at tens of $\mathrm{keV}$

Fig. 4 A few examples of extreme Balzars, i.e. those having the most powerful jets, larger black hole masses and more luminous accretion disks [16]
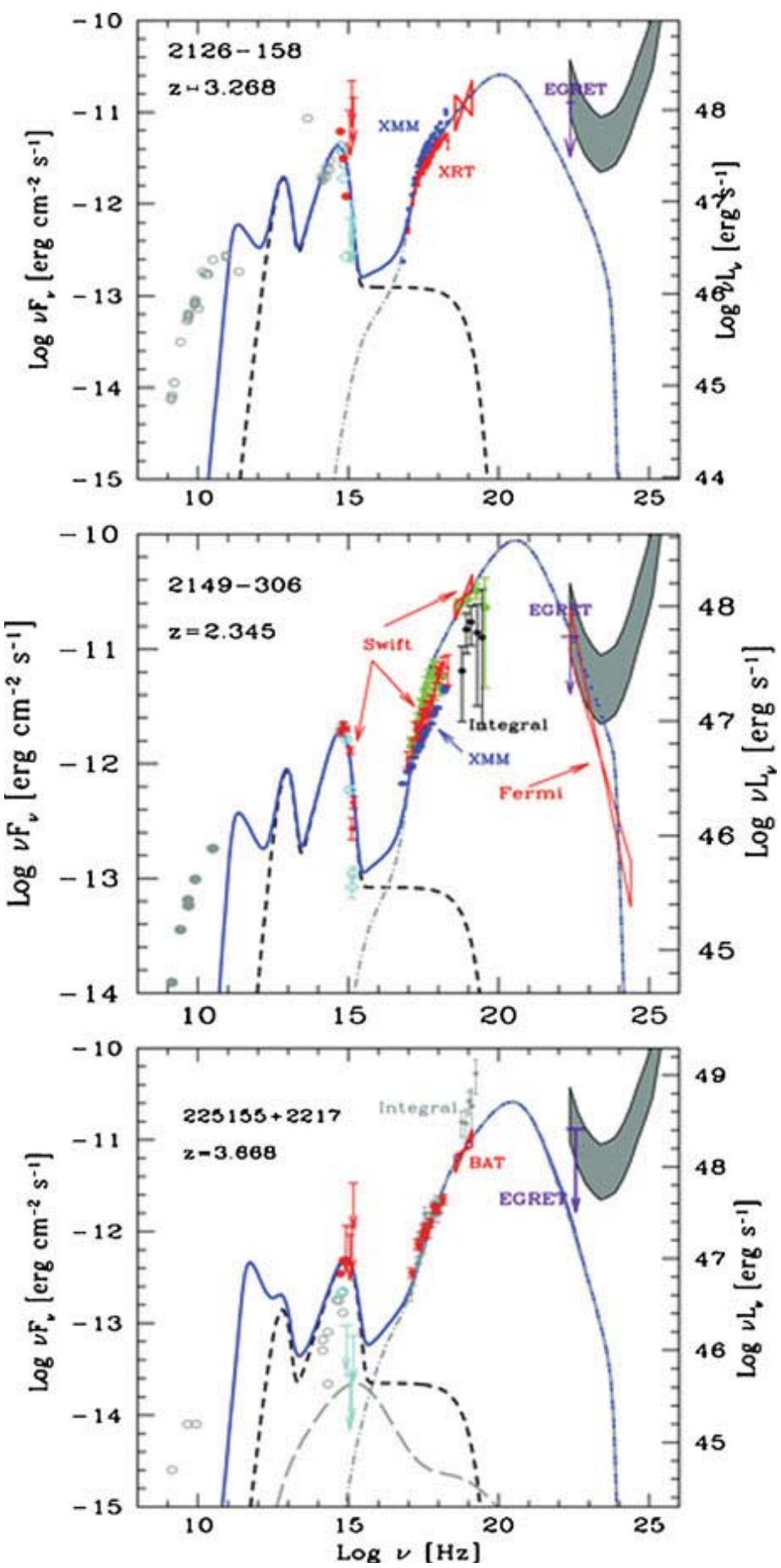
or more. At the other end of the sequence are located sources with the most powerful jets, larger black hole masses and more luminous accretion disks: the Compton peak of their spectral energy distribution falls in the $\mathrm{MeV}$ sub-MeV region (see Fig. 4 for a few examples). Thus the hard X-ray selection provides a new perspective into jet dominated sources and new insights into the physics connecting jet and disk emission. X-ray polarisation studies would also provide detailed and original physical information on the jet physics and geometry and will be crucial to understand the frequency dependence of blazar polarisation, an issue which is so far limited to sporadic frequency coverage in the radio and optical bands.

\subsubsection{Accretion on all scales: from stellar mass black holes to AGN}

Up to now the existence of various spectral states was clearly established only in X-ray binaries. There is evidence, based on variability properties and radio emission, that similar state transitions exist also in AGN and ULXs. So far it seems that the hard X-ray spectra of AGN do not match the classification of stellar mass black holes. For instance, AGNs that are supposed to be in the hard state based on their Eddington ratios appear to have very soft spectra $(\Gamma>2)$. While the power law index distribution of AGN has been well investigated [5], observational results on the reflection and cut-off energy (Ec) have so far been limited due to the poor statistical quality of measurements above 10-20 keV. However, a recent analysis of a complete sample of type 1 AGN detected by INTEGRAL give evidence for a mean cut-off at around $100 \mathrm{keV}$ with most objects displaying $\mathrm{E}_{c}$ in the range 50-150 keV (Molina, M., et al., in preparation). Therefore a leap in sensitivity in the range $50-150 \mathrm{keV}$ appears crucial. Precise cut-off energy measurements together with determination of other AGN parameters such as reflection luminosity, obscuration, redshift etc, will clarify the working mode of the central engine. Beyond that, by analogy with X-ray binaries, it is important to look for a dichotomy in AGN spectral states (soft/steep /no cut-off versus hard/flat/ 100-200 keV cut-off), to identify the main emission mechanism. It can also be tested whether narrow line Seyfert 1 galaxies (thought to be at an early stage of evolution) have the same cut-off than classical Seyfert $1 \mathrm{AGN}$, with clear implication on the cosmic evolution of SMBH. Only an accurate determination of the characteristics of the high energy spectral shape in a statistical significant and complete sample of accretion dominated AGN can answer to these and other questions thus allowing a fundamental step in our understanding of the physics of growing black holes.

With a sensitivity 50 to 100 better that INTEGRAL, we can detect a source as bright as 3C273 up to $\mathrm{z}$ around 1 thus making evolution studies of AGN spectral properties feasible. Occasionally, brighter sources can be detected up to $\mathrm{z}$ a few like IGR J22517+2218 $(z=3.7)$ seen by INTEGRAL up to $100 \mathrm{keV}$ (400 keV in the source rest frame, [2]). One can then probe reflection and energy cut-off in various classes of active galaxies in the local Universe and up to the age where quasar activity was at its peak; or probe the Compton peak in 
Blazar SED (Spectral Energy Distribution) from local BL Lacs to more distant Flat Spectrum Radio QSOs. Studying AGN properties over a broad range of redshifts will also provide a new insight into the evolution of SMBH.

\subsubsection{Obscured AGN and the hard X-ray cosmic background radiation}

The high energy extragalactic background emission, which covers an extremely wide range from about $1 \mathrm{keV}$ up to $100 \mathrm{GeV}$, is believed to originate from the summed contributions of different types of AGNs. However, in the hard $\mathrm{X}$-ray bandpass the situation is not so clear, since most models predict a number of low- to-intermediate luminosity AGN at the peak of the accretion activity $(\mathrm{z} \sim 1.5-2.5)$ higher by a factor of a few relative to $\mathrm{X}$-ray observations. The disagreement is not surprising, given that present $\mathrm{X}$-ray surveys miss most Compton Thick (CT) AGN [9], which may well be common at highz. Good hard X-rays (>10-20 keV) sensitivity and spatial resolution are thus mandatory if one wants to uncover a substantial fraction of the missing AGN population and get a precise characterization of their spectral energy distribution at $\mathrm{E}>100 \mathrm{keV}$.

Only a dedicated hard X-ray instrument will be capable to provide robust constraints on the broad-band high energy emission and finally settle the issue of quantifying the missing (CT or new) AGN population.

\subsection{Physics of neutron stars}

Observing neutron stars has also proved to be an efficient tool to investigate the properties of matter in the most extreme environments. Neutron stars are also the most magnetized objects we know. Similar to black holes, neutron stars in binary systems can accrete matter from their companion. Such objects are sometimes called accreting X-ray or accretion-powered pulsars because the accreting material of these systems emits large amounts of X-ray radiation just before it reaches the neutron star surface, providing us with unique information about its immediate vicinity, and the properties matter in extreme gravitational and magnetic fields. The main challenges are to measure the intensity and structure of the magnetic field around neutron stars, to understand the physics of accretion near their surface, and to model the particle acceleration in these sources. In contrast to black holes, the accreting material cannot go through the magnetic field lines generated inside the neutron star and is instead channelled to the poles where it hits the surface almost in free fall $(v=0.7 \mathrm{c})$, on a quite small area. There, a shock forms (see Fig. 5) and the infalling material is heated to very high temperatures $\left(\mathrm{T}=10^{7} \mathrm{~K}\right)$.

The qualitative picture of this accretion mechanism is now well understood and different spectral signatures have been detected. Diagnostically the most important spectral features are the so-called cyclotron resonance scattering features (CRSFs), which result from the quantization of the electron cyclotron motion around the field lines, appear as absorption lines, and arise only in the strong field regime of a few $10^{12} \mathrm{G}$; Figs. 5 and 6 . 
Fig. 5 Structure of the accretion column at a polar cap (from [3])

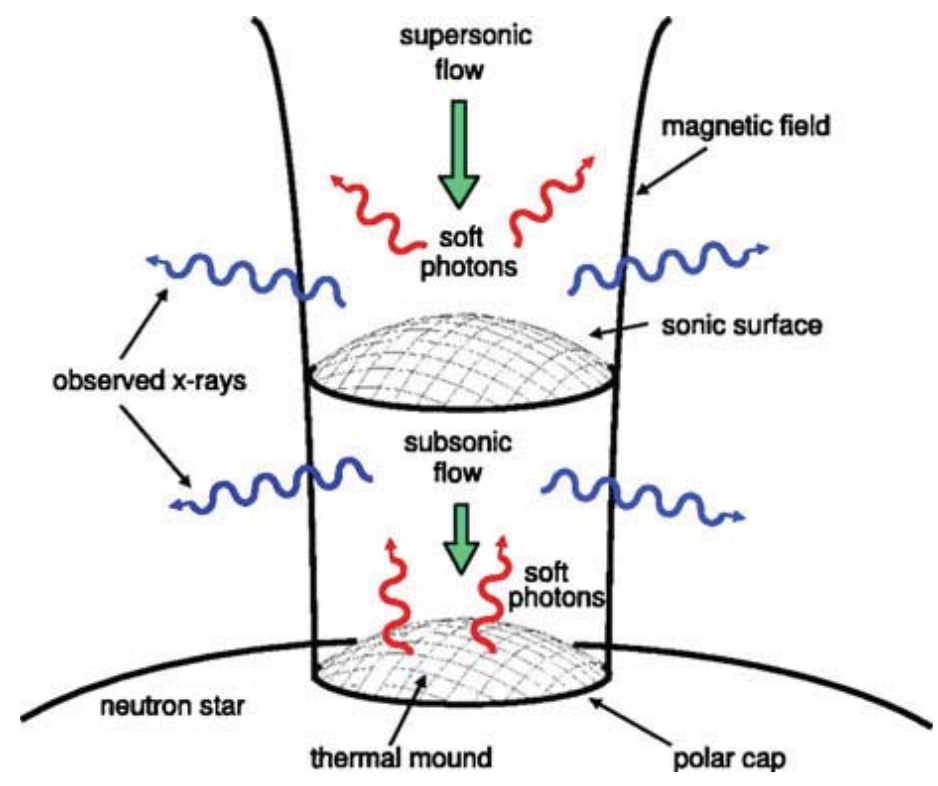

Simple, homogeneous emission models produce a fundamental cyclotron line at energy $\mathrm{E} \_0=11.6 \mathrm{keV}\left(\mathrm{B} / 10^{12} \mathrm{G}\right)$ and harmonics at energies $\mathrm{E} \_\mathrm{n}=$ nE_0. Thus, the determination of the line energies provides us with a unique method to measure the magnetic field intensity directly. The typical energy of the fundamental line lies in the 10-100 keV range, showing direct evidence for magnetic field of about $10^{12}-10^{13} \mathrm{G}$ (see, e.g., [22]). Recent work has shown that, in addition to the line energy, the detailed line profile (depth, shape...) also depends on the properties of the emission region (temperature, density, magnetic field...) and is affected by the accretion geometry [26]. A good sensitivity and energy resolution in $10-100 \mathrm{keV}$ is required to increase the sample size and possibly detect stronger magnetic fields with lines at higher energies.

Fig. 6 Pulsar 4U 0115+63 spectrum, with bremsstrahlung (dashed line), iron line (dotted-dotteddotted-dashed line), and synchrotron emission continuum with absorption features (dotted-dashed line) [13]

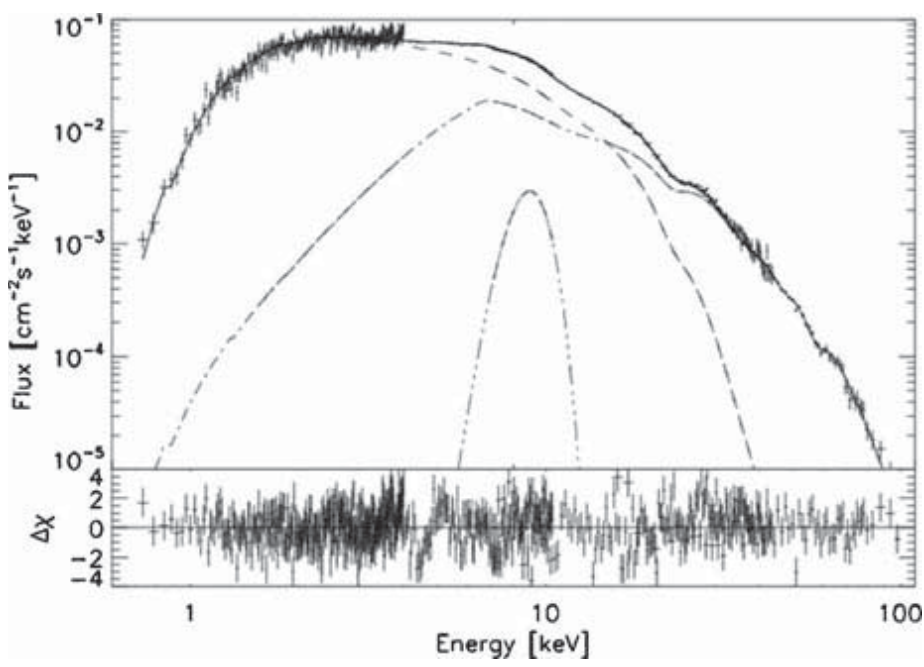


Time variations at various time scales are inherent to accreting neutron stars. Spectral evolution on the week-long outburst timescales is present, including variation of the cyclotron line energy [31]. One big step forward will be to perform phase resolved spectroscopy at these timescales, which would require a time accuracy much smaller than $1 \mathrm{~ms}$

More generally, all pulsars are also known to produce strong hard X-ray and gamma ray emission. When not dominated by accretion emission, this very high energy emission is thought to originate from relativistic particles accelerated in the magnetosphere. However, the location of the acceleration region is unknown, and different models have been proposed where the emission region is very close to the neutron star surface in the accretion column (polar cap models) or further away in the magnetosphere (outer gap models). Here again, timing and pulse resolved spectroscopy at short time scales $(<1 \mathrm{~s})$ are necessary in order to discriminate these models.

Lastly, in addition to spectral signatures, strong polarisation is also expected at all wavelengths. Polarized emission at optical and radio wavelengths has been observed for years, but only recently was X-ray polarisation detected in one pulsar (the Crab nebula, [10]), with a polarisation level of several tens of percent. Polarisation measures might thus open a new window on the physics of accreting neutron stars.

\section{The PheniX telescope}

PheniX is based upon two co-aligned hard X-ray telescopes operating in parallel. These telescopes have a focal length of $40 \mathrm{~m}$ obtained with an extensible mast. The instrument concept is based on two grazing incidence focusing optical modules located "inside" the spacecraft, an extensible mast $40 \mathrm{~m}$ long, and two shielded high energy imaging detectors on top of the mast, see Fig. 7.

The two identical detector assemblies, also called focal plane assemblies, FPA, on top of the mast compose the "Detector Module". Each detector assembly has the following main elements:

- Cold box containing the planar Ge detector and the pre-amplifiers (PA)

- $90 \mathrm{~K}$ passive radiator and cold rod cooling the Ge detector

- $210 \mathrm{~K}$ passive radiator and heat pipes cooling the cold box and the PAs

- Telescopic aperture stops

- The anticoincidence (AC) detector surrounding the cold box

- Analog and digital front end electronics for the GeD and the ACD

- A payload computer

The mast and the optical metrology between the mirrors and the focal plane are considered part of the service module and described in the relevant chapters. 


\subsection{Instrumental concept}

Since the launch of the Einstein satellite, focusing optics in a Wolter I configuration have been "commonly" used to increase the collection area and thus the sensitivity of instruments (XMM, Chandra, Suzaku) in the X-ray domain $(0.1$ to $15 \mathrm{keV})$. We propose to extend this concept in the hard X-ray range up to $200 \mathrm{keV}$ together with a large collecting area. This leads to three main requirements:

- As the wavelength associated with the photon decreases with the energy, the maximum grazing angle drastically reduces. This difficulty is overcome by an increase of the telescope focal length to $40 \mathrm{~m}$.

- As energetic photons have a higher penetrating power, the mirror coating has to be more "efficient" in order to maintain a good reflection coefficient when the photon energy increases. This is achieved by using dedicated depth-graded multi-layer coating technique.

- The increase of effective area is obtained through a large number of mirror shells. This is now feasible using light weight mirror shells such as slumped glass mirror substrate.

The focal plane of the instruments has to detect photons in the 1-200 keV energy range with a good spatial accuracy and a very good energy resolution. In addition, we aim for a single detector and polarimetry measurement capabilities. We propose to use the state of the art semi conductor detector: a double sided strip germanium detector, in a planar configuration, operating around $90 \mathrm{~K}$. Thanks to its unique physical properties: very small band gap,

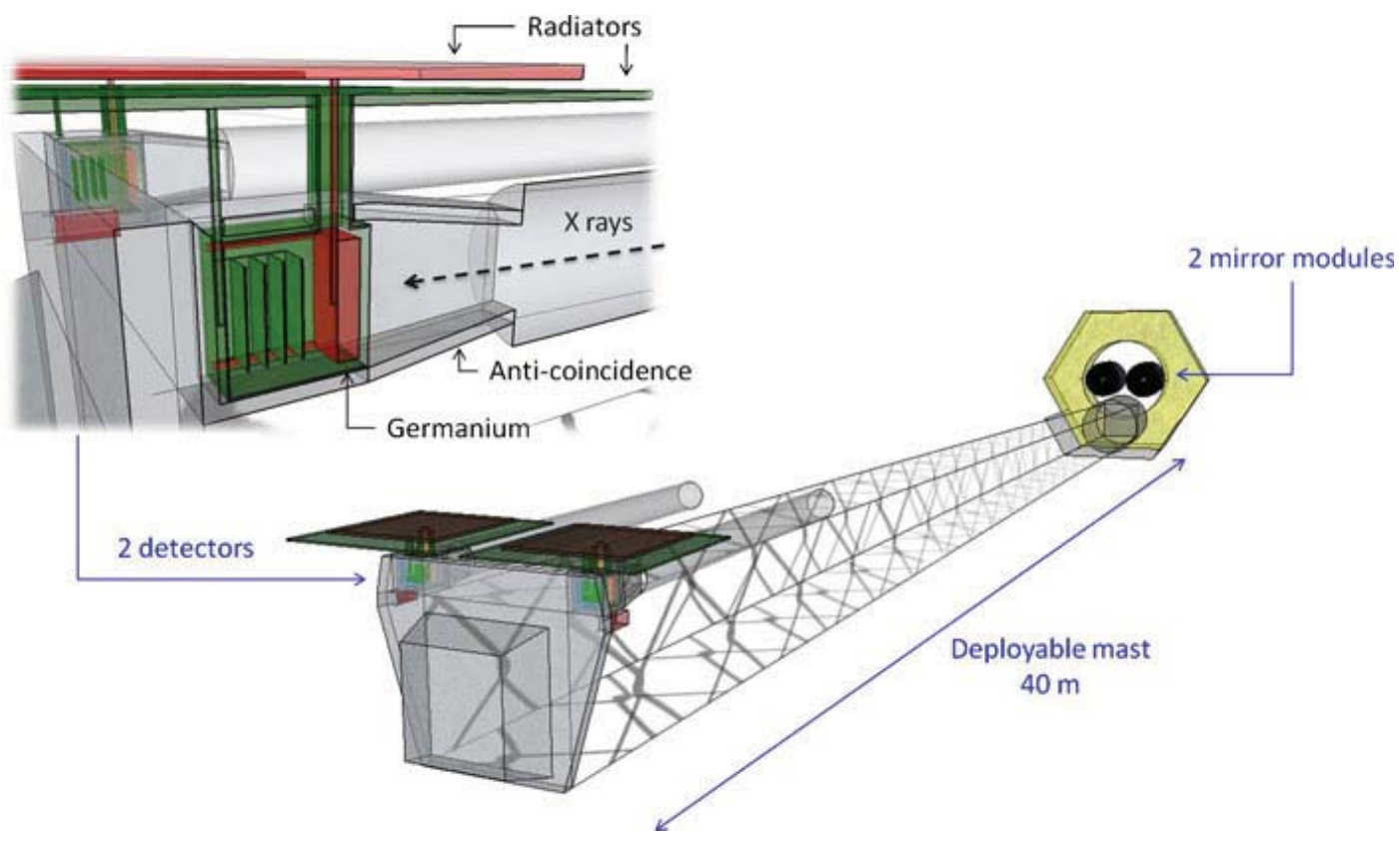

Fig. 7 Instrumental concept 
high velocity of the charge carriers, low leakage current and perfect recovery of irradiation damage by annealing, this detector will be able to cover the full energy range of the instrument with the required performance.

The detectors sensitivity could be affected by photons not coming from the mirror field of view and by protons. These photons and charged particles induce a background noise in the detectors and increase the transmitted data rate from satellite to the Earth. In order to ensure an as low as possible background, the whole system will be surrounded by an active shielding named anticoincidence. This active shielding will give a fast signal when traversed by a charged particle. As this charged particle could generate background signal in the X-ray detector (directly, by going also through the imaging detector, or indirectly by generating photons through the surrounding matter), this fast signal will be used as a veto signal for the imaging detector acquisition. This system will be complemented by a passive shielding used to reduce the contribution of the cosmic X-ray background in the aperture.

The two telescopes operating in parallel increase the collection area and thus will improve the sensitivity. Moreover, they provide a very simple redundancy scheme as we have two independent systems in hot redundancy (Fig. 7).

\subsection{Instrument conceptual design and key characteristics}

\subsubsection{The optics}

PheniX telescope relies on two co-aligned mirror modules in a Wolter-I configuration. The parameters of each mirror module are:

\begin{tabular}{ll}
\hline Focal length & $40 \mathrm{~m}$ \\
Mirrors & 260 \\
Radius min & $0.05 \mathrm{~m}$ \\
Radius max & $0.31 \mathrm{~m}$ \\
Shell length & $0.5 \mathrm{~m}$ \\
Total mirror length & $1 \mathrm{~m}$ \\
Field of view (Aeff $>50 \%$ on axis) & $6 \mathrm{arcmin}$ \\
Coating A (depth graded multilayer) & $100 \mathrm{Pt} / \mathrm{C}$ bilayers \\
Coating B (depth graded multilayer) & $1100 \mathrm{Co} / \mathrm{C}$ bilayers \\
Mass (slumped glass hypothesis) & $175 \mathrm{~kg}$ \\
\hline
\end{tabular}

To focus high energy photons (above $100 \mathrm{keV}$ ), we need mirrors capable of very high grazing incidences which lead to very small collecting areas with respect to the mirror length. To overcome this, we use longer mirrors $(1 \mathrm{~m})$ than typical ones ( $0.6 \mathrm{~m}$ for XMM).

The mass has been estimated from slumped glass technology ("The Nuclear Spectroscopic Telescope Array (NuSTAR): Optics Overview and Current Status" [17]) but silicon pore optics can also be considered [8]. In order to increase the reflectivity at high energy, we have to use an adapted depth graded 
multilayer (DGML). The standard option (coating A) is composed of $\mathrm{W} / \mathrm{C}$ or $\mathrm{Pt} / \mathrm{C}$ bilayers. The total (2 mirror modules) effective area using this coating (Fig. 8: blue line) exhibits an important edge at $78 \mathrm{keV}$ (Pt K-edge absorption). To overcome this efficiency drop, we have explored alternative coatings using materials with lower absorption, such as described by Belloti and Windt [4]. They have produced a prototype Co/C DGML showing excellent X-ray performance beyond $80 \mathrm{keV}$ together with a factor 3 reduction in surface roughness $(\sigma \sim 0.2 \mathrm{~nm})$, low film stress and excellent temporal stability. The red line in Fig. 8 shows the effective area using this coating. The nominal design for PheniX is this $\mathrm{Co} / \mathrm{C}$ coating (B), while the $\mathrm{Pt} / \mathrm{C}$ coating (A) is a backup.

The angular resolution of the mirror modules will strongly rely on the assembly and alignment of the mirrors. The expected angular resolution is $20 \operatorname{arcsec}$ HEW at $10 \mathrm{keV}$.

This design has been developed using the numerical tool DynamiX [7]. This numerical tool aim at the simulation of grazing incidence telescopes accounting for each mirror default (roughness, figure, assembly, alignment), multilayer reflection (depth graded layers, interface roughness), detector response (X-ray interactions) and for the deformation of the telescope structure.

\subsubsection{The "Cold Box" sub-system}

The germanium detector needs to be cooled to $90 \mathrm{~K}$. The temperature stability has to be better than $1 \mathrm{~K}$ per day. During the annealing operation, it has to be heated to $378 \mathrm{~K}$ for a few days. In order to reduce the electronic noise, the preamplifiers will be operated at around $210 \mathrm{~K}$. The required stability has to be better than $0.1 \mathrm{~K}$. These requirements are inherited from SPI/Integral in-orbit experience.

The thermal concept (Fig. 9) is similar (with a scaling factor) to SPI/Integral: A cold-box at $210 \mathrm{~K}$ contains the preamplifier block $(10 \times 10 \times 10 \mathrm{~cm})$ and the

Fig. 8 The total PheniX simulated effective area for the two modules (Red: $\mathrm{Co} / \mathrm{C}$, Blue: $\mathrm{Pt} / \mathrm{C}$ )

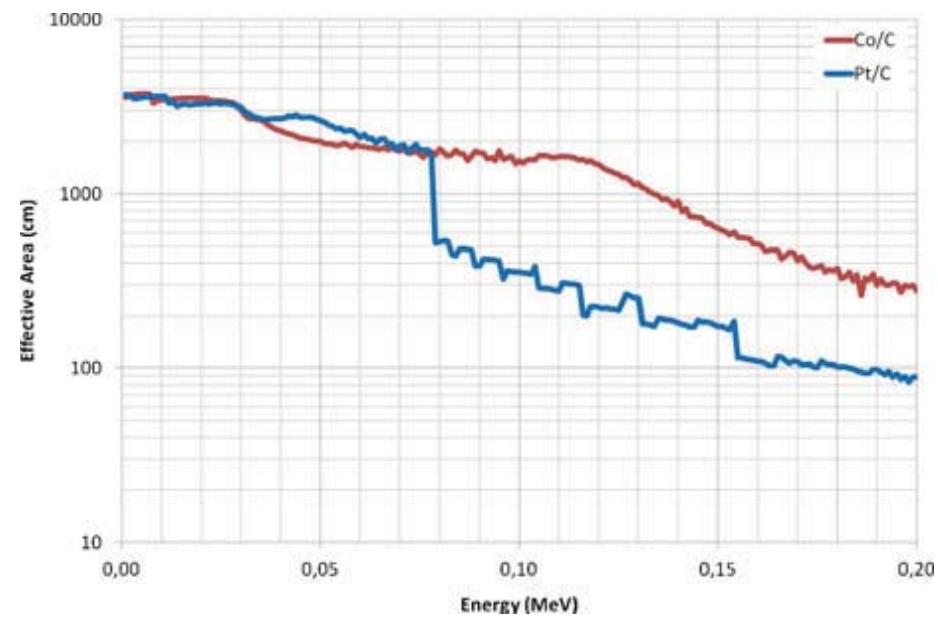


Fig. 9 The detector assembly and the thermal concept

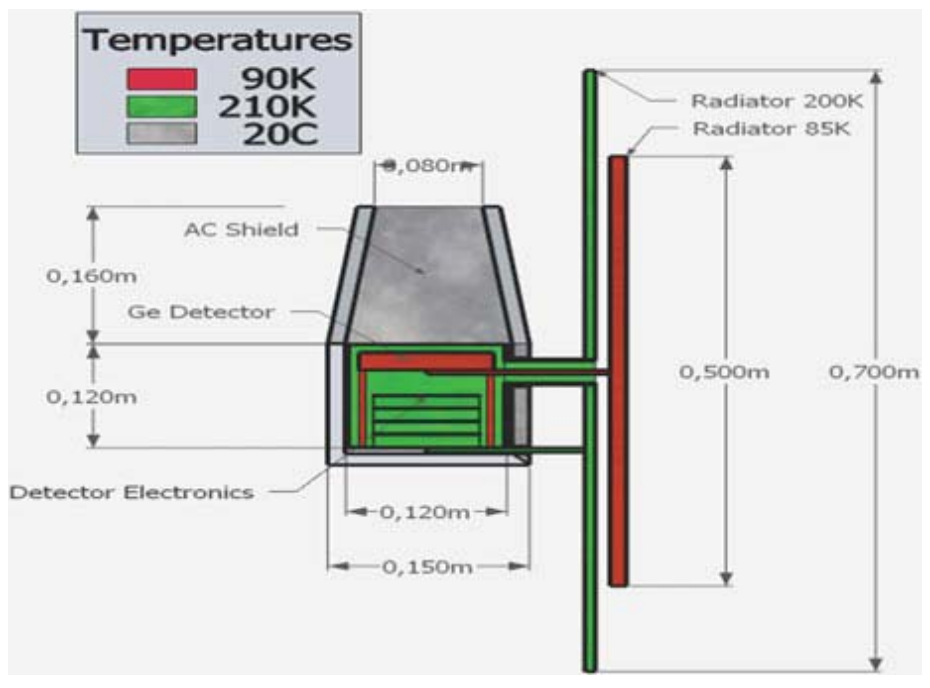

Germanium detector $(10 \times 10 \times 2 \mathrm{~cm}$ box $)$ at $90 \mathrm{~K}$. The two thermal stages concept allows: (a) to operate the preamplifier at low temperature and (b) to decrease the thermal leakage at $90 \mathrm{~K}$. Taking into account a nominal orbit at $\mathrm{L} 2$, the thermal design proposed here is based on a complete passive cooling system:

- For the $90 \mathrm{~K}$ stage: we computed a total thermal load of $500 \mathrm{~mW}$. This can be evacuated by a radiator of $1800 \mathrm{~cm}^{2}$ at $85 \mathrm{~K}$.

- For the $210 \mathrm{~K}$ stage: we computed $20 \mathrm{~W}$ in total to evacuate. This will be achieved by a radiator of $2300 \mathrm{~cm}^{2}$ via heat pipes.

For the Ge detector annealing, we need to heat the detector up to $378 \mathrm{~K}$. To achieve this we will need either a thermal switch to disconnect the $85 \mathrm{~K}$ radiator or to install enough heating power. For reference, SPI/Integral has already

Fig. 10 The detector assembly

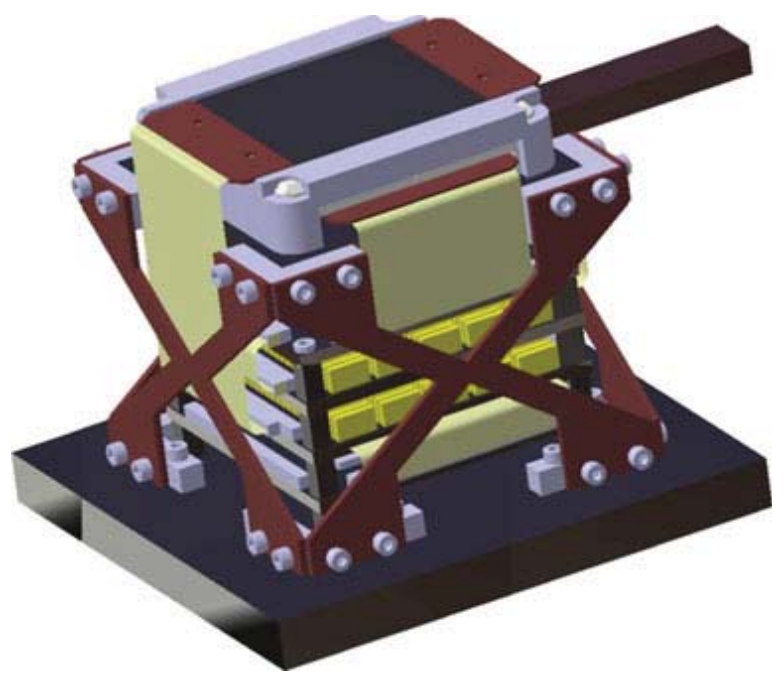


flawlessly undergone 17 annealing cycles $(80 \mathrm{~K}-373 \mathrm{~K})$ since the beginning of the mission.

\subsubsection{The detector and associated pulse shape processing}

The detector is a high purity Germanium detector used in a planar configuration. The physical size of the detector is $90 \times 90 \mathrm{~mm}$ and $15 \mathrm{~mm}$ thick, while the detection area is $80 \times 80 \mathrm{~mm}$ (Fig. 10).

Each face of the detector is segmented with $0.5 \mathrm{~mm}$ pitch strip contacts. The strips are orthogonal between the two faces. We thus have 160 strips on each face.

When a photon interacts with the germanium electron/holes pairs are created and collected by the electrodes. A simple localization scheme is to find, for each direction (each detector face), the strip which gives the maximum signal. The localization accuracy is then equal to the pitch of the strips (Fig. 11). A more complex scheme will also use the signal measured on the adjacent strips. Then interpolation technique will allow an improvement of the spatial resolution allowing a reduction of the number of analysis channels.

The charges resulting from an interaction are collected on the two faces of the detector. The analysis of the charge collection times will provide the depth of the interaction with accuracy of 1-2 mm. This depth measurement capability leads to two important features: (a) the internal background of the detector will be reduced as the detector "thickness" can be adjusted to the photon energy, (b) the detector becomes 3 dimensional allowing, in case of a Compton interaction, an improvement of the polarisation measurement of the primary photon.

Fig. 11 Strip detector, signal acquisition

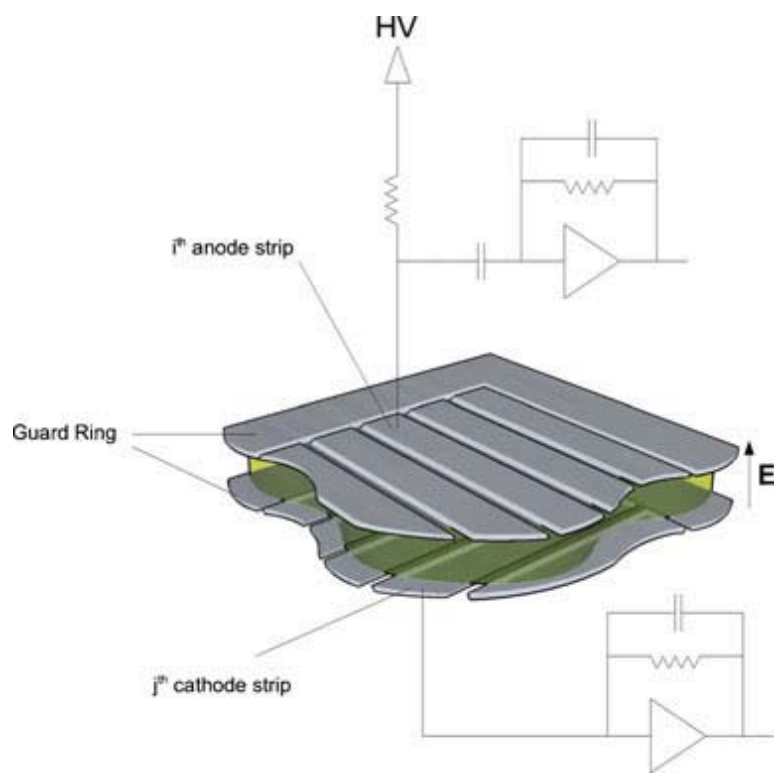


The performance of the detector will be obtained thanks to a precise and complex analysis of the signals produced. In order to have a high degree of flexibility all the pulses will be digitalized after the preamplifier. All the parameters (energy, position, depth) are determined by digital electronics (Fig. 12).

The signal from each strip goes to a charge preamplifier which is located in the $210 \mathrm{~K}$ cold box providing an important noise reduction for the preamplifier. The output of the preamplifiers, after amplification, will be continuously digitalized.

The signals, after digitalization, are analyzed by a fast digital electronics (FPGA/DSP) that will extract the physical parameters of the interaction: energy, coordinates of the interaction, precise time stamping, coincidences.

Each strip has its own analysis channel, preamplifier fast-amplifier ADC. Thanks to the relatively low number of channels, this is feasible with "conventional" existing radiation-hard electronic components. In summary: the detector is a double sided strip germanium detector, this detector is built from a single monolithic crystal and will have following characteristics:

- Useful area: $8 \times 8 \mathrm{~cm}$-thickness: $15 \mathrm{~mm}$-Mass: $650 \mathrm{gr}$

- Electrodes: thin electrodes, 160 on each face

- Operating temperature: $90 \mathrm{~K}$ will need regular annealing at $378 \mathrm{~K}$

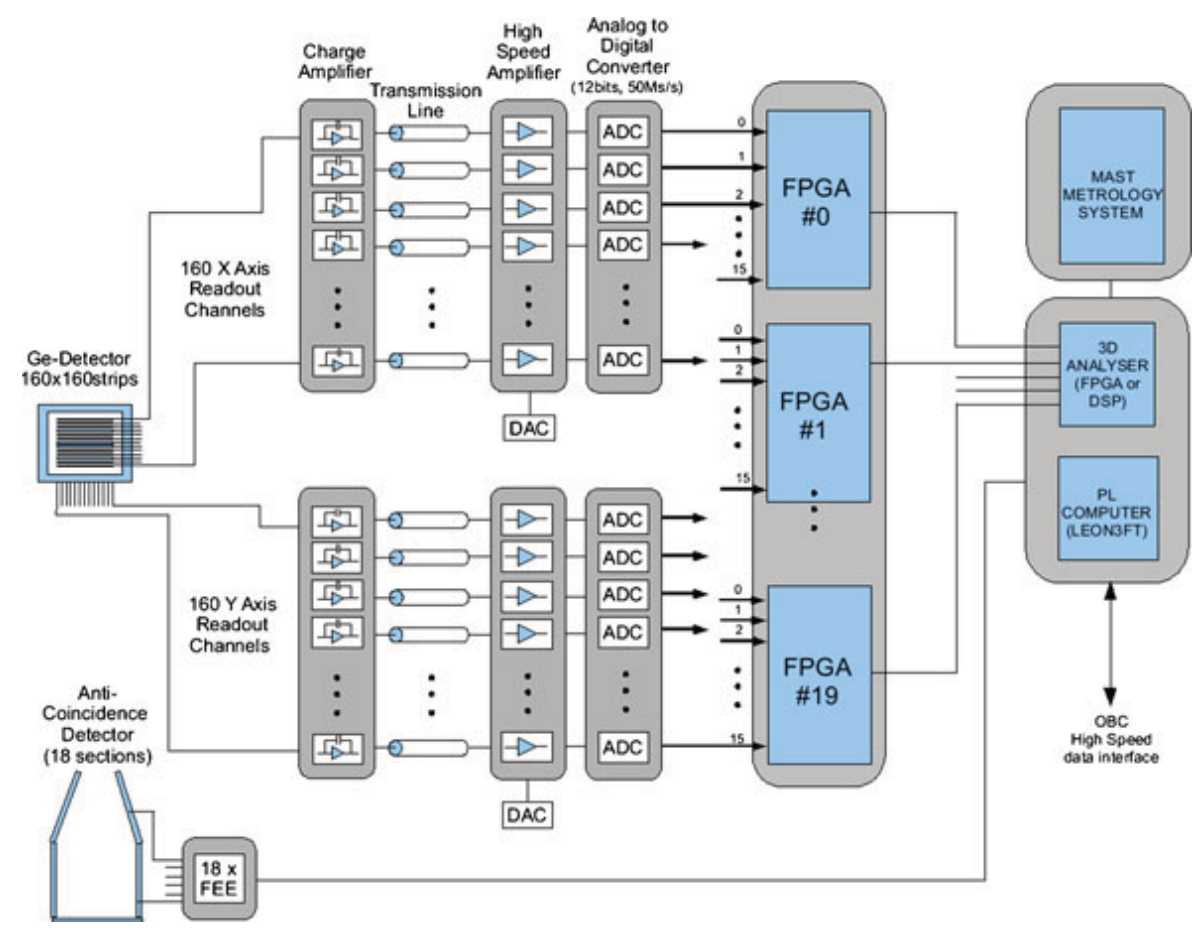

Fig. 12 PheniX electronic design 
- Spatial resolution $0.5 \mathrm{~mm}-$ Depth resolution: $1 \mathrm{~mm}$

- Energy resolution: $150 \mathrm{eV} @ 6 \mathrm{keV}-400 \mathrm{eV} @ 100 \mathrm{keV}$

- Energy range: $1-200 \mathrm{keV}$ Time tagging: better than $100 \mathrm{~ns}$

- Efficiency: better that $80 \%$ in the whole range.

- Counting rate without pileup: better than 100000 counts/s

\subsubsection{Payload on board computer and data handling}

The two focal planes will have independent data processing chains. Each chain will have its own Payload Computer interfacing with the Ge detector 3D analyzer, the AC subsystem and the platform On-Board Computer (OBC) and the mass memory (MM).

The Payload Computer will manage the various high speed data links, perform the anti-coincidence algorithm and generate the science data.

The Payload Computer and Mass Memory could be based on the same design developed during phase-B for the Simbol-X mission, using currently available LEON processors.

\subsubsection{The anticoincidence}

Inorganic scintillators, such as $\mathrm{BGO}$, CsI, or $\mathrm{NaI}$, besides marking proton passages, could stop photons up to $200 \mathrm{keV}$. Among the possible crystals, we have chosen $\mathrm{NaI}$ as it is the easiest to implement and it can reach low energy threshold required for the particle and photon rejection. Among photodetectors, we have chosen APD as they are light and also easy to implement mechanically. The total mass for AC system including its electronics is $19.1 \mathrm{~kg}$, the power consumption is estimated to $5 \mathrm{~W}$.

In order for the detector to stay within the maximum internal background limit due to the particle environment of $10^{-5} \mathrm{ph} / \mathrm{cm}^{2} / \mathrm{s} / \mathrm{keV}$ as required, the anticoincidence main specifications are the following:

- Fully hermetic to photons of energy lower than $200 \mathrm{keV}$ outside the FOV.

- Expected event number: around 100 counts per second from photons; 3500 counts/s from protons.

- AC time tagging resolution : < $100 \mathrm{~ns}$. Tagging efficiency: $>99 \%$

- Low energy threshold : $30 \mathrm{keV}$

\subsubsection{Passive aperture stop}

With the long focal length of PheniX it is impractical/impossible to extend a collimator up to the mirrors. With the open angle required for the active shield much larger than the mirror modules, much of the field of view is open to the background. We propose the use of a number of aperture stops that efficiently shield the instrument from the isotropic background in a design similar to that of NuSTAR. The aperture stops consist of a series of $2 \mathrm{~mm}$ thick lead annuli 


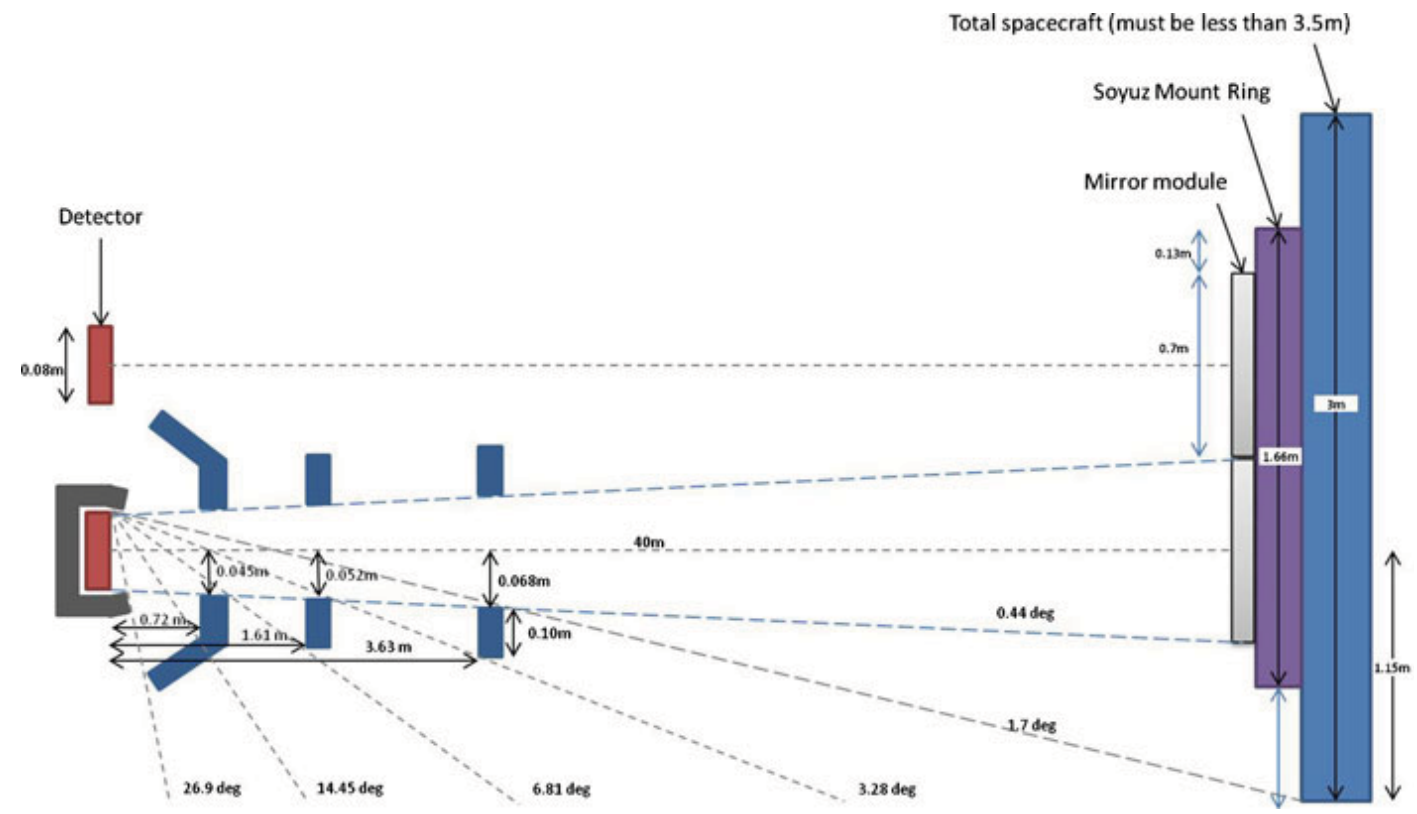

Fig. 13 passive aperture stop

that will extend out in front of the detector on poles as the mast extends, blocking over $85 \%$ of the background at $200 \mathrm{keV}$ (Figs. 7 and 13).

\subsection{Performance assessment with respect to science objectives}

\subsubsection{Angular resolution}

The angular resolution of the telescope depends on the mirror modules angular resolution and on the detectors alignment measurement.

The angular resolution of the mirror modules will strongly rely on the assembly and alignment of the mirrors. The slumped glass technology, using Wolter-I conical approximation, is able to provide a mirror module with an angular resolution around 40 arcsec HEW (cf. NuSTAR). But using a WolterI configuration and improving the mirrors assembly/alignment accuracy, we expect an angular resolution less than 20 arcsec at low energies (ref IXO slumped) for PheniX.

As we want to push this concept up to $200 \mathrm{keV}$, the micro-roughness of the mirrors responsible of X-ray scattering (XRS) becomes significant. XRS increases the focal spot size with energy. Using the design with $\mathrm{Co} / \mathrm{C}$ coating will reduce this component thanks to its low surface roughness $(\sigma \sim 0.2 \mathrm{~nm})$. The expected angular resolution is 80 arcsec at $100 \mathrm{keV}$. The internal deformation of the telescope during an observation will degrade the angular resolution. We need to measure the internal alignment between the mirrors and the detector to correct the position of each detected photon. As the pixel pitch of the detectors is $0.5 \mathrm{~mm}$, we need to measure the detector alignment with an accuracy of the same level. 


\subsubsection{Background and sensitivity}

Being situated at the L2 Lagrangian point drastically reduces the Earth albedo and trapped particle components of the background. The instrumental background is reduced further by minimising the passive materiel around the detectors to reduce the delayed background components. Combining the low background and the large effective area gives PheniX excellent continuum sensitivity (Fig. 8). Due to the detail of the high-energy background environment being unknown at L2, the sensitivity has also been calculated for a background 5 times greater than that predicted by simulations. This sensitivity curve does not take into account the reduction in background that can be achieved with depth of interaction measurements in the detector which can reduce the background by 2 . Even taking the larger background, PheniX provides a significant increase of sensitivity compared to existing missions and surpasses their sensitivity for a 10 times longer observation (Fig. 14).

\subsubsection{Polarimetry performance}

Usually for Compton polarimetry the energy range where Compton scatters dominate is considered. However, Compton scatters continue to occur at lower energies, just at a much reduced rate than the number that interact through the Photoelectric effect. Many sources in the X/Gamma-ray regime such as the Crab exhibit a power law spectrum and therefore emit many more photons at low energies. This is enhanced further by the large effective area PheniX provides at lower energies. The Compton scattering cross section also shows a

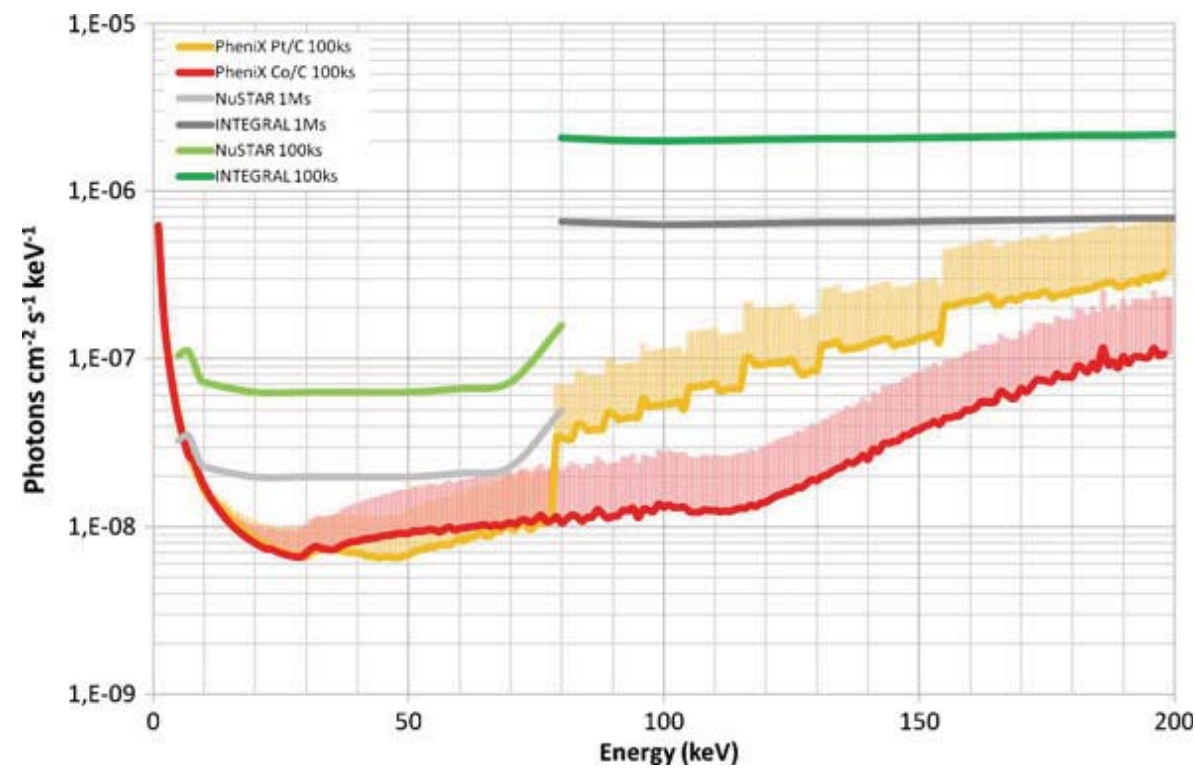

Fig. 14 The continuum sensitivity osf PheniX for $100 \mathrm{ks}$ observations, based on a $3 \sigma$ detection with $\Delta \mathrm{E} / \mathrm{E}=0.5$ and an internal background of $\sim 1 \mathrm{e}^{-5} \mathrm{c} \mathrm{cm}^{-2} \mathrm{~s}^{-1} \mathrm{keV}^{-1}$. The shaded area demonstrates the sensitivity if the background is greater at $\sim 5 \mathrm{e}^{-5} \mathrm{c} \mathrm{cm}^{-2} \mathrm{~s}^{-1} \mathrm{keV}^{-1}$. For comparison the INTEGRAL and NuSTAR sensitivities are plotted for $100 \mathrm{ks}$ and $1 \mathrm{Ms}$ 
great anisotropy at lower energies, and it is this anisotropy that we use to detect the photon polarisation. Combining these facts allows the energy ranges that Compton polarimetry is used to be pushed down to $50 \mathrm{keV}$ (Fig. 15).

The Q-factor, defined as the ratio of the parallel and perpendicular components of the scattered distribution, is a useful figure of merit for polarimeters. The single germanium block of PheniX can be pixelated very finely using the strip readout technique, allowing the tuning of the pixel size to best record the low energy Compton scatters. Polarimeters measuring the electron scatter from the photoelectric effect, very fine position sensitivity is required to measure the electron path as close to the initial interaction site as possible to avoid the loss of signal due to multiple scatters. In the PheniX detector the pixel size is tuned so that the initial scatter occurs in one pixel and then the photon photo-ionises in a second and providing a high Q-factor (measured for experimentally at $\sim 200 \mathrm{keV}$ to be $\sim 0.6$, see $[18,20,30])$.

The minimum detectable polarisation percentage (MDP\%) based purely on photon statistics as defined by Weisskopf et al. [34] provides another useful figure of merit and an indication of the polarisation performance of the instrument. PheniX will be capable of detecting $1 \%$ polarisation in $10^{5} \mathrm{~s}$ for the brightest and in $10^{6} \mathrm{~s}$ for a $100 \mathrm{mCrab}$ source.

The design of the detector plane will keep systematics to a minimum. However, it is important to consider how the mirrors affect the incident source flux. Almeida and Pillet [1] analysed the X-ray optics of AXAF and showed that in theory there will little reduction in polarisation for any number of scatters and that grazing-incidence telescopes are extremely well suited for polarimetry. Recently, Katsuta et al. [19] measured the polarisation effect for $\mathrm{Pt} / \mathrm{C}$ mirror section to be used for ASTRO-H and NuStar at $30 \mathrm{keV}$ and showed that it will retain the source polarisation to better than $1 \%$. It is

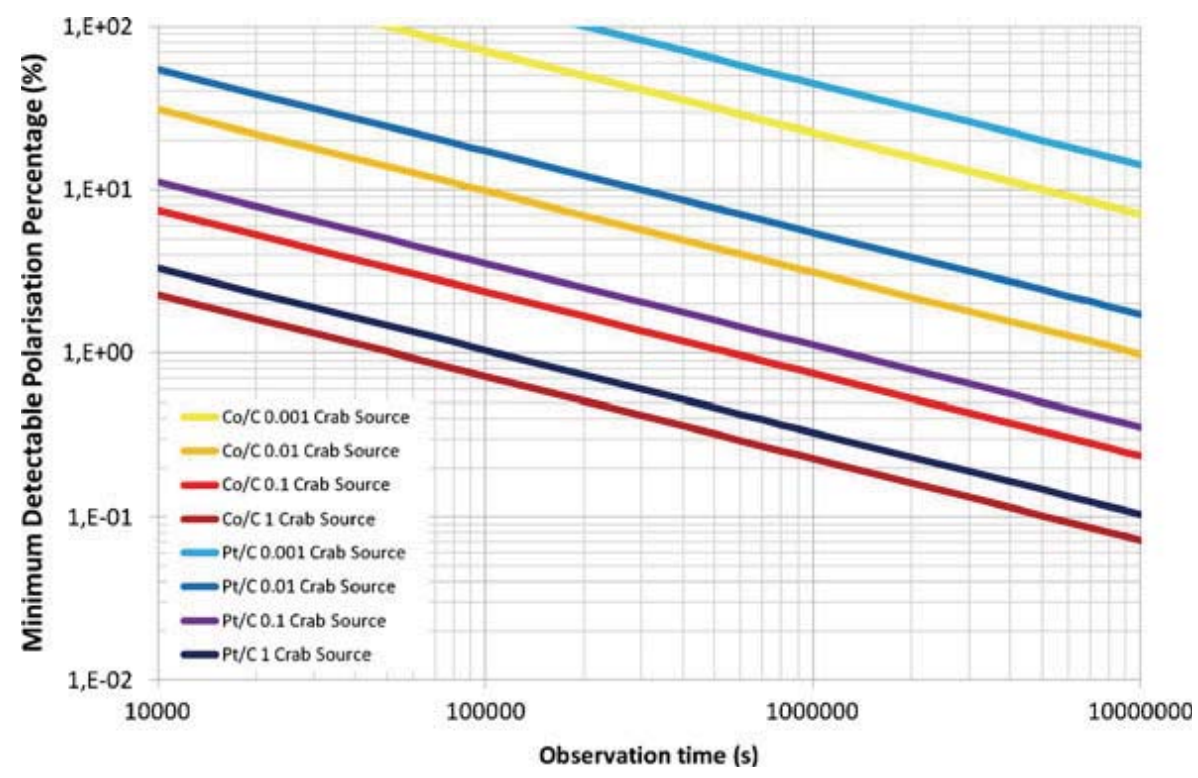

Fig. 15 The PheniX minimum detectable polarisation (50-200 keV) for different observation times and different source strengths and mirror coatings 
anticipated that the effect of the mirrors can be calibrated out prior to launch. However, how the polarisation is affected by the mirrors is an area that will need to be fully explored in the full design process.

2.4 Resources: mass, volume, power, on board data handling and telemetry

\subsubsection{Mass and power}

The power needed for each detector module including the on-board computer has been estimated to $130 \mathrm{~W}$, leading to a total of $260 \mathrm{~W}$ power needed.

The mass of each detector module has been estimated to $65 \mathrm{~kg}$ (including the ACD the collimator, cooling system and computer) leading to a total mass of $130 \mathrm{~kg}$ for the whole detection assemblies.

\subsubsection{On-board data handling and telemetry}

A typical PheniX observation plan could last one week and encompass a few sources, of which, some could be very bright and produce a lot of data.

Based on the effective area and the detector energy range and assuming, as sizing case, an observation plan of 1 Crab source for 1 day and fainter sources for 6 days producing about the same amount of data, it is estimated to generate 15 GB of data to download per week, per detector. Assuming one ground contact per week dedicated to download the data at high bit rate, the size of the Mass Memory in the Payload Computer should be at least 30 GB.

It must be noted that the high performance payload computer implementing science data reduction algorithms on-board, the large Mass Memory storage, together with the intended operating modes/configurability will allow setting the instrument for the different target source types, optimizing at any time the science data return to the available downlink telemetry bandwidth.

\subsubsection{Communication, pointing, alignment, timing requirements}

With the typical observation plan, it is estimated to generate $30 \mathrm{~GB}$ of science data to download per week. A RF link in X band with high bit rate for TM download has been proposed. The proposed operations concept foresees the scheduling of one $15 \mathrm{~m}$ ground station for a short pass every day, during working hours, for command and control operations and one or two long passes per week to download the data using a $35 \mathrm{~m}$ ground station.

The spacecraft pointing has to be stable because of the mirrors vignetting. With an offset of 20 arcsec of the pointing axis with respect to the target, the effective area decrease is $50 \mathrm{~cm}^{2}$. A pointing control of 20 arcsec is thus required. The absolute pointing has to be known at each moment within a few arcsec accuracy for the image reconstruction.

The optical modules have to be co-aligned with accuracy better than 20 arcsec. Their optical axes have to be known with respect to the star tracker axis with an accuracy of 5 arcsec. The lateral displacement of the detector payload 
with respect to the star tracker has to be known at each moment with an accuracy of $0.5 \mathrm{~mm}$.

After the mast deployment the detector payload position has to be adjusted in the focal plane. This adjustment with be done via stepper motors located at the base of the mast. The required precision of the alignment is within a few $\mathrm{mm}$. This calibration procedure will be repeated as required. The focal length, i.e. the length of the mast, has to be known with an accuracy of $+/-10 \mathrm{~cm}$.

\subsubsection{Orbit requirements}

Due to the proposed configuration of the spacecraft and payload, to simplify their design, an orbit with reduced thermal flux variations and perturbations is required. A libration orbit around a Lagrange point would be very beneficial for the spacecraft and would allow a smooth and already proven operation concept. Here we summarize the main advantages of this orbit for the PheniX mission:

- No atmospheric drag and gravity effects: This is favorable for the mast solution. Very low thrust consumption $(0,2 \mathrm{~m} / \mathrm{s}$ DeltaV/year in Herschel) for attitude control and station keeping is expected.

- Very good thermal stability, it allows no eclipses: This is favorable for the passive cooling design proposed for the $80 \mathrm{~K}$ stage of the focal plane and for thermal stability of the mirrors.

- One ground antenna could ensure coverage for several hours per day.

- ESA has experience to design LEOP, transfer phases and routine operations in this orbit (Herschel and Planck).

\subsubsection{Current heritage}

Mirrors The mirrors modules are the most challenging part of PheniX development. While our study is based on slumped glass technology used in NuSTAR instrument [17], significant differences exist the total length of the mirrors is increased to $1 \mathrm{~m}$, the number of nested shells is 260 , we aim for an improved angular resolution $\left(20^{\prime \prime}\right.$ at $\left.10 \mathrm{keV}\right)$. However, we have kept the same substrate density and shell thickness. In addition, we aim to use a $\mathrm{Co} / \mathrm{C}$ bilayer coating optimized at higher energies $(150 \mathrm{keV})$ that exhibits very good performances [4]. We continue to consider the standard $\mathrm{Pt} / \mathrm{C}$ or $\mathrm{W} / \mathrm{C}$ coating as a backup solution. In order to attain the PheniX objectives a significant, but feasible, effort has to be conducted.

Mast ABLE technology has considerable experience in mast systems for space application; we thus consider the components for ADAM mast covering our needs are currently available. Note that several ADAM masts of $60 \mathrm{~m}$ length have already flown for the STRM program. See http://www.aecable.com/Booms/adam.html 
Germanium detectors have been used in space since 35 years in a variety of experiments (HEAO-3, TGRS, RHESSI, mars Odyssey, SPI/Integral) for high resolution spectroscopy. Last mission, SPI/Integral, has demonstrated that, while continuously irradiated, an annealing process restores their original performance even after 8 years in space. Strip planar germanium with electrodes pitch down to 250 microns detectors have been realized for ground applications, e.g. see Stohlker et al. [30] or Rossi et al. [28].

\section{The all sky monitor (ASM)}

The ASM telescope is based upon the coded mask technique already used in the GRANAT, Integral and Swift missions. Hard X-ray sources cast a shadow on the detector through the spherical mask, which enables to retrieve on Earth the source direction. The shape of the mask and the design of the mask aperture are optimized to have a large field of view of up to $60-70^{\circ}$ half-angle away from the telescope axis with a moderate angular resolution. The whole system is rotating, in order to avoid source confusions and improve the imaging performance of the telescope. The detector is a matrix which will register the $\mathrm{X}, \mathrm{Y}$ impact position, energy and arrival time of each detected photon.

The telescope will consist in $16 \mathrm{CdTe}$ cameras derived from the "Caliste 256" cameras, formerly developed for the Simbol-X French-Italian project. Each Caliste module is equipped with 256 pixels $(16 \times 16)$ monolithic Schottky CdTe diode, $10 \mathrm{~mm}$ by side, $2 \mathrm{~mm}$ thick and houses 8 multichannel analog front-end chips (IDeF-X). Pixels pitch is $625 \mu \mathrm{m}$; the area of a single camera is $1 \mathrm{~cm}^{2}$.

These cameras will be protected from the diffuse X-ray background by a passive shielding made of $1 \mathrm{~mm}$ of Tantalum. A spherical coded mask, with a spiral-shaped aperture, is placed above the Caliste cameras, providing a field of nearly 4 sr (see Fig. 16).

The main performances of this All Sky Monitor will be the following:

\begin{tabular}{ll}
\hline Energy range & $5-200 \mathrm{keV}$. \\
Field of view & $4 \mathrm{sr}\left(\sim 70^{\circ}\right.$ opening angle $)$. \\
Energy resolution & $1 \mathrm{keV} @ 60 \mathrm{keV}$. \\
Temporal resolution & $50 \mu \mathrm{s}$. \\
Angular Resolution & $\sim 40 \operatorname{arcmin}$. \\
Sensitivity & $\sim 50 \mathrm{mCrab}(5 \sigma, 10 \mathrm{ks}, 5-200 \mathrm{keV})$. \\
Point source location accuracy & $\sim 2 \operatorname{arcmin} .(10 \sigma$ source $)$ \\
\hline
\end{tabular}

Total mass has been estimated to $5 \mathrm{~kg}$ and the power consumption to $3.5 \mathrm{~W}$. 
Fig. 16 view of a Caliste camera (left) and drawing of the spherical mask (right)

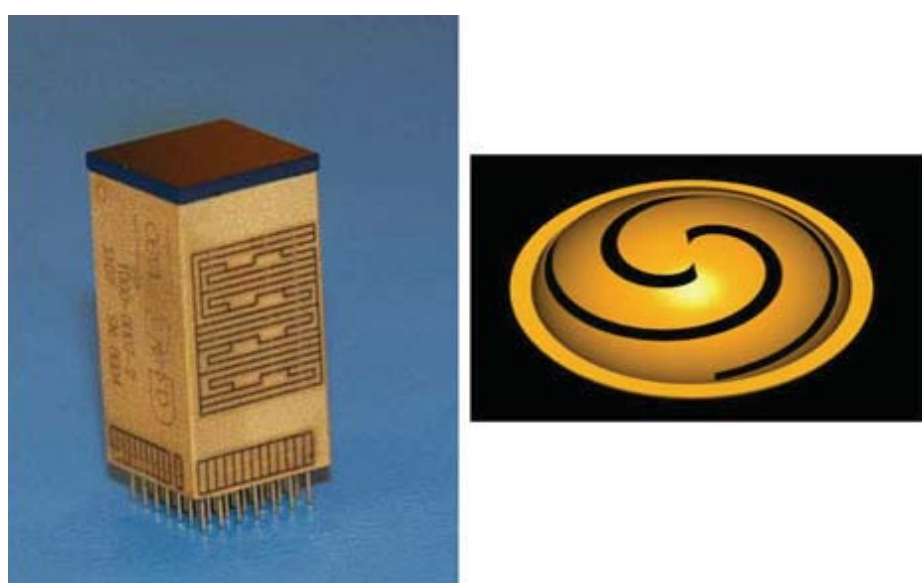

\section{Summary and conclusion}

We have shown that major progresses on all of the presented issues (and others) could be achieved with a very sensitive broad band (1-200 keV) hard-X-ray spectro/polarimeter with good spectral and spatial resolutions. The timing accuracy, mainly driven by pulsar science but also crucial for timing studies in X-ray binaries, should reach 50-100 $\mu$ s.

Comparing to similar operating (INTEGRAL, SUZAKU) or planned (Nustar, Astro-H) missions, the main improvement would be to have a unique instrument covering a broad energy range with much improved sensitivity up to $200 \mathrm{keV}$ thanks to a new coating and longer focal length. The 1$200 \mathrm{keV}$ band pass allows one to constrain several distinct spectral components that must be measured and studied simultaneously to obtain original and unquestionable results on the physics of compact objects.

A sensitivity around a few $10^{-8}$ photons $/ \mathrm{cm}$. s. $\mathrm{keV}$ at $100 \mathrm{keV}$ will represent a gain of a factor greater than 50 relatively to INTEGRAL one, while at $20 \mathrm{keV}$, it ensures a gain by a factor of 5 over Nustar expectations. Together with an effective area similar to $\mathrm{XMM}$ at a few $\mathrm{keV}$, this provides an interesting trade-off over the global bandpass. This means that we will not only detect Galactic sources in their quiescent state (i.e. $10^{-7}$ LEdd) but also be able to determine precisely their spectral shape, test the presence of a cutoff or other characteristics linked to the emission mechanisms. Similarly, we will catch all the AGNs currently detected below $10 \mathrm{keV}$ but missed above that, with the opportunity to study their hard X-ray emission, and most importantly, their cut-off energy.

Such sensitivity makes sense only if we are able to disentangle all the detected sources in a same field of view. We aim at a spatial resolution better than 30" which should be enough to image SNRs and resolve the numerous hard X-ray sources at the Galactic centre. 
PheniX detector features a very good energy resolution which is crucial for physics based on line emissions. This encompasses nucleosynthesis thematic (through decay chains of heavy elements, for us ${ }^{44} \mathrm{Ti}$ ) and the Iron $\mathrm{K} \alpha$ line emitted by reflection on cold matter structures in the innermost regions around compact objects. The observed line position and shape include a lot of information on the emitting sites environment characteristics.

Finally, it appears that cleaver detector design can now provide sensitive $\mathrm{X}$-ray polarimetry. Such a capability will indubitably open a radically new window on X-ray sources. While we will offer performances similar to dedicated instrument below $40 \mathrm{keV}$, the domain above $40 \mathrm{keV}$ will be open for the first time, at the same level of sensitivity. This information will complement the spectral and timing data and be a key to substantial progress.

Summary of the PheniX mission characteristics.

\begin{tabular}{lll}
\hline Parameters & Requirement & Drivers \\
\hline Energy range & $1-200 \mathrm{keV}$ & All \\
Spectral resolution @ 7 keV & $150 \mathrm{eV}$ & Fe line(XRB, AGN) \\
( $\Delta \mathrm{E} / \mathrm{E}$ FWHM) $100 \mathrm{keV}$ & $0.5 \mathrm{keV}$ & ${ }^{44} \mathrm{Ti}$ lines \\
Angular resolution & Better than $30^{\prime \prime}$ & SNR, Galactic Cen \\
Sensitivity @ 7 keV & $410^{-8} \mathrm{ph} / \mathrm{cm} . \mathrm{s} . \mathrm{keV}$ & All \\
$3 \sigma, 10^{5} \mathrm{ks} @ 100 \mathrm{keV}$ & $210^{-8} \mathrm{ph} / \mathrm{cm} . \mathrm{s} . \mathrm{keV}$ & \\
For $\Delta \mathrm{E} / \mathrm{E}=0.5 @ 200 \mathrm{keV}$ & $1510^{-8} \mathrm{ph} / \mathrm{cm} . \mathrm{s} . \mathrm{keV}$ & \\
Polarimetry MDP & $1-\mathrm{a} \mathrm{few} \%(0.1 \mathrm{Crab})$ & Pulsars,XRB,AGNs \\
Time accuracy & Better than $100 \mu \mathrm{s}$ & Pulsars \\
Min count rate without pile-up & A few Crab $\left(2.10^{5} \mathrm{c} / \mathrm{s}\right)$ & Bright sources \\
\hline
\end{tabular}

In addition to this main telescope, a hard X-ray monitor has been considered. Its main role will be to detect any important event in the hard X-ray sky. It must have some capacity of localization in order to provide the position of the target to point. The characteristics of PheniX will not only enrich our understanding of high energy production at all scales in our Galaxy, but also in the universe in general. The unprecedented sensitivity will open a large area of discoveries, probing the faintest hard sources for the first time. Finally, PheniX will provide a new window onto matter under extreme conditions.

\section{References}

1. Almeida, Pillet: Polarizing properties of grazing-incidence X-ray mirrors: comment. Appl. Opt. 32, 4231 (1993)

2. Bassani, L., et al.: INTEGRAL/IBIS 7-year all-sky hard X-ray survey II. Catalog of sources. ApJ 669, L1 (2007)

3. Becker, P.A., Wolff, M.T.: Thermal and bulk comptonization in accretion-powered X-ray pulsars. ApJ 654, 435 (2007)

4. Bellotti, J.A., Windt, D.L.: Depth-graded Co/C multilayers prepared by reactive sputtering. SPIE 7437, 38 (2009) 
5. Bianchi, et al.: CAIXA: a catalogue of AGN in the XMM-Newton archive. I. Spectral analysis. A\&A 495, 421 (2009)

6. Blandford, R.D., Znajek, R.L.: Electromagnetic extraction of energy from Kerr black holes. MNRAS 179, 433 (1977)

7. Chauvin, M., Roques, J.P.: DynamiX, numerical tool for design of next-generation X-ray telescopes. Appl. Opt. 49, 4077 (2010)

8. Collon, M.J., et al.: Performance of silicon pore optics. SPIE 7011, 7011 (2008)

9. Comastri, A.: ASSL 348, 245 (2004)

10. Dean, A.J., et al.: Polarized gamma-ray emission from the crab. Science 321, 1183 (2008)

11. Droulans, R., et al.: Variability and spectral modeling of the hard X-ray emission of GX 339-4 in a bright low/hard state. ApJ 717, 1022 (2010)

12. Fabian, A.C.: XMM-Newton and broad iron lines. AN 329, 155 (2008)

13. Ferrigno, C., et al.: Study of the accreting pulsar $4 \mathrm{U} 0115+63$ using a bulk and thermal comptonization model. A\&A 498, 825 (2009)

14. Ghisellini, G., et al.: A theoretical unifying scheme for gamma-ray bright blazars. MNRAS 301, 451 (1998)

15. Ghisellini, G.: Blazars in hard X-rays. AIP Conf. Proc. 1126, 131 (2009)

16. Ghisellini, G., et al.: Chasing the heaviest black holes of jetted active galactic nuclei. MNRAS 405, 387 (2010)

17. Hailey, C.J., et al.: The Nuclear Spectroscopic Telescope Array (NuSTAR): optics overview and current status. SPIE 7732, 28 (2010)

18. Inderhees, et al.: Spectroscopy, imaging and compton-scatter polarimetry with a germanium strip detector. IEEE NSS(1995) $\mathbf{1 6 5}$ (1996)

19. Katsuta, et al.: Evaluation of polarization characteristics of multilayer mirror for hard X-ray observation of astrophysical objects. Nucl. Instrum. Methods A 603, 393 (2009)

20. Kroeger, et al.: Gamma ray polarimetry using a position sensitive germanium detector. Nucl. Instrum. Methods A 436, 165 (1999)

21. Makishima, K., et al.: Suzaku results on cygnus X-1 in the low/hard state. PASJ 60, 585 (2008)

22. Mihara, et al.: Cyclotron observations of binary X-ray pulsars. Prog. Theor. Phys. Suppl. 169, 19 (2007)

23. Miniutti, G., Fabian, A.C.: A light bending model for the X-ray temporal and spectral properties of accreting black holes. MNRAS 349, 1435 (2004)

24. Narayan, R., Yi, I.: Advection-dominated accretion: a self-similar solution. ApJ 428, L13 (1994)

25. Narayan, R., et al.: Estimating the spins of stellar-mass black holes by fitting their continuum spectra. AIPC 968, 265 (2008)

26. Nishimura, O.: Formation mechanism for broad and shallow profiles of cyclotron lines in accreting X-ray pulsars. ApJ 672, 1127 (2008)

27. Petrucci, P.O., et al.: Unveiling the broad band X-ray continuum and iron line complex in Mrk 841. A\&A 470, 889 (2007)

28. Rossi, et al.: X-ray response of germanium microstrip detectors with energy and position resolution. Nucl. Instrum. Methods A 392, 264 (1997)

29. Schnittman, J.D., Krolik, J.H.: X-ray polarization from accreting black holes: coronal emission. ApJ 712, 908 (2010)

30. Stohlker, et al.: A 2D position sensitive germanium detector for spectroscopy and polarimetry of high-energetic X-rays. JPhCS 58, 411 (2007)

31. Tsygankov, S.S., et al.: Completing the puzzle of the 2004-2005 outburst in V0332+53: the brightening phase included. MNRAS 401, 1628 (2010)

32. Turner, T.J., Miller, L.: X-ray absorption and reflection in active galactic nuclei. A\&ARv 17, 47 (2009)

33. Volonteri, M.: Evolution of massive black hole spins. Proc of the Conference Accretion and Ejection in AGNs. arXiv:1002.3827 (2010)

34. Weisskopf, et al.: The prospects for X-ray polarimetry and its potential use for understanding neutron stars. 363rd Heraeus Seminar in Bad Honef, Germany. arXiv:astro-ph/0611483 (2006) 